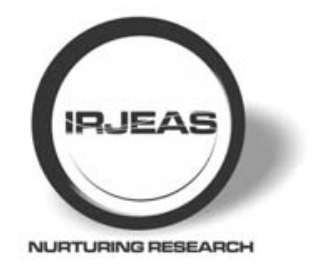

INTERNATIONAL RESEARCH JOURNAL

OF

ENGINEERING \& APPLIED SCIENCES

\title{
MULTI-STORY AND INTELLIGENT CAR PARKING SYSTEM
}

\author{
*Aaradhna Soni ${ }^{1}$ \\ Asst. Professor, Department of Electrical \& Electronics Engineering, Sage University, Bhopal, India
}

*Corresponding author - aaradhna.s@sageuniversity.edu.in

\begin{abstract}
The proposed work implements a 8 bit controller based multi-storey car parking system. This work aimed to make the current vehicle parking technique increasingly advantageous, easy to use, programmed and least human mediation. The central part of this system is a 8 bitmicrocontroller which takes care of parking and exit of vehicles. There are totally 3 floors (floor 0,1 and 2) each containing 8 slots (slot 0 to slot 7 ) for parking of cars. Cars are carried to the available free slots in any floor by means of 2 motors controlled by the P89v51rd2(8051 microcontroller). LCD displays the user instructions, messages and status of the system at different conditions. Keypad is provided for entering parking or taking out option. One IR sensor is used to detect the floor number by sensing the IR signal from the IR transmitters located in each floor. The user is provided with the facility of prebooking or reservation of slots using GSM (Global System for Mobile Communications).
\end{abstract}

Keywords-Sensors, slots, automation, GSM.

Manuscript Received: 16 December 2021, Received in Revised form: 10 January 2022, Accepted: 15 January 2022

DOI - 10.55083/irjeas.2022.v10i1003

(C) 2022 The author. This is an open access article under the CC BY license. (https://creativecommons.org/licenses/by/4.0/)

\section{INTRODUCTION}

Nowdays in numerous multiplex frameworks there is a serious issue for vehicle leaving framework. There are numerous paths for vehicle leaving, so to leave a vehicle one needs to search for the all paths. Additionally there is a great deal of men work required for this procedure for which there is parcel of speculation. So the need is to build up a framework which shows legitimately which slots are empty in any path at a given point of time.The system includes a framework including infrared transmitter and recipient in each path and a LED and LCD show outside the vehicle leaving door. So the individual entering parking region can see the LCD display and can choose which path to enter or to leave the vehicle. Expectedly, vehicle leaving frameworks does not have any wise checking framework. Parking slots are managed by individuals. All vehicles go into the parking region and search for leaving or entering. Once in a while it makes blockage. Condition become more regrettable when there are numerous stopping paths and every path have different stopping spaces. Utilising of automation framework for vehicle entry and exit will decrease the human endeavours. Display unit is introduced on passage of parking area which will show through LCD for all Parking spaces. Empty slot is shown in the LCD display unit which will be helpful and appropriate for the users. As of late, inquire about has utilized vehicle-to vehicle and vehicle-to-framework association with the help of different remote system advancements, for example, radio frequency identification (RFID), Zigbee, remote wreckage organizes, and the Internet. This investigation planned to give data about adjacent parking spots for the driver and to reserve a spot minute sooner utilizing upheld gadgets, for example, cell phones or tablet PCs. Moreover, the administrations utilize the ID of every vehicle in booking a parking spot. Be that as it may, the current canny stopping framework does not give a by and large ideal arrangement in finding an accessible parking spot, does not take care of the issue of burden adjusting, does not give monetary advantage, and does not get ready for vehicle refusal administration.

Currently, the regular strategy for finding a parking spot is manual where the driver as a rule finds a space in the road through karma and experience. This procedure requires some serious energy and exertion and may prompt the most pessimistic scenario of 
neglecting to discover any parking spot on the off chance that the driver is driving in a city with high vehicle thickness. The option is to discover a predefined vehicle leave with high limit. In any case, this isn't an ideal arrangement in light of the fact that the vehicle park could more often than not be far from the client goal.

This proposed work includes a framework including infrared transmitter and beneficiary in each path and a LED and LCD show outside the vehicle leaving entryway. So the individual entering leaving zone can see the LED showcase and can choose which path to enter in order to leave the vehicle. Show unit is introduced on passage of parking garage which will indicate LEDs for all Parking space and for all stopping paths. Void space is demonstrated by the individualglowing LED and appropriate display on LCD. The person has the facility for prebooking of slot from home or workplace with ease , this is possible with GSM module.

The customary leaving frameworks, for example, staggered or multi-store vehicle leaving frameworks (nonrobotized), robot vehicle leaving frameworks, computerised staggered vehicle leaving frameworks and so forth have been actualized on an enormous scale. In any case, these frameworks have a noteworthy hindrance of vast space utilisation which is effectively wiped out with the utilisation of "Programmed vehicle leaving system". In the advancementof traffic the executives frameworks, a savvy leaving framework was made to decrease the expense of procuring individuals and for ideal utilisation of assets for vehicle leaveproprietors. This motivates us to provide identication using RFID with and pre-booking facility using GSM with additional features.

\section{RELATED WORK}

These days parking structures have ended up being dreary and needs some portion of work to manage and care for it. Thesestopping zones are difficult to utilize and don't give data as for openness of free spaces. Various researchers have added to this issue and formalized with various strategies to all themore promptly improve the parking structure to serve the prerequisites.

In [1], the creator proposed savvy stopping reservation framework utilizing short message services (SMS), for that he utilizes Global System for Mobile(GSM) with microcontroller to improves security. The ZigBee systemis utilised alongside the GSM module for stopping the board and reservation. The creator utilises Global Positioning System(GPS) and android stage to demonstrate accessible parking spots. In any case, booking for the equivalent isn't accessible .

In the work propsed by [2], it utilises Artificial Intelligence (AI) systems to process pictures, which perceives the leaving involved just by vehicles. The framework gives direction pictures towards the alloted spaces, subsequently making it shrewd.Inter integrated circuit (I2C) convention is utilized alongside vehicle leaving framework $(\mathrm{CPF})$ to dole out radio recurrence distinguishing proof (RFID) to every vehicle which will be utilized to recognize vehicle left over anopening. Variable message screen(VMS) demonstrates vehicle left over a given floor. The framework allocates and saves an ideal parking spot dependent on drivers cost work that consolidates closeness to goal and stopping cost. Driver demand preparing focus (DRPC) gives foundation to vehicle (I2V) correspondence for doling out and saving parking spots utilizing smart parking allocation center (SPARC).

The author of [3] utilises wide point camera as a sensor which recognise just free parking spots and records them. These records are then used to appoint parking spot to the approaching client. Canny Transport System (ITS) and Electronic toll accumulation (ETC) utilising optical character recognition (OCR) makes a record for all entering vehicle. This makes label less passage for all vehicles in the parking garage, however it doesn't allocate a space to the client. A general OCR calculation isn't accessible, making it hard to make said records.

A mechanical carport utilizing Bluetooth which would be utilized to completely mechanize the situation of a vehicle in the opening without the guide of the driver been proposed by the authors of [4]. The framework consequentlychecks the one of a kind enlistment number put away in the Bluetooth chip to check if the new vehicle ought to be left. This framework is a vertical leaving game plan for the vehicles with sensors that affirm position of the vehicle. Different sensors are utilized to affirm that there are no travelers left in the vehicles and after that the framework moves the vehicle to capacity territory utilizing rack and pinion ( $\mathrm{RaP}$ ) instrument. The creator proposes updated framework to the above mentioned, which is conveyed with radio frequency identification (RFID) to authenticate at the gate 
management service (GMS) to dole out a complete space. The framework gives an extra element to screen parking areaover the web .

\section{METHODOLOGY}

The proposed work is based on the embedded systems with GSM module.The system uses a central micro-controller with a unique RFID card and display the messages to the user with the help of LCD display. The user can book the slots which are available.The working can be illustrated using the block diagram.

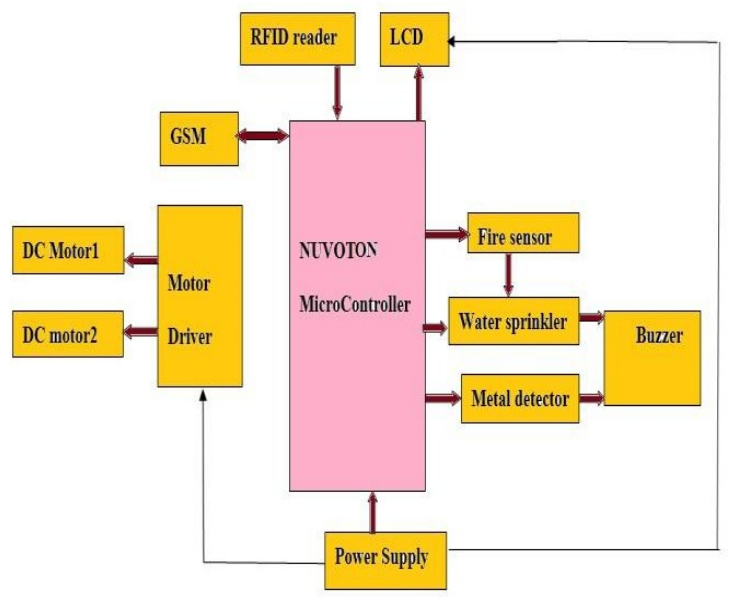

Figure 1: Block diagram of the proposed system.

The block diagram is shown Figure 1. The user has to swipe his smart card to park his car. The controller searches for the available free slot by searching the parking records stored in its memory. If free slot is not available, it displays thismessage on the LCD. Otherwise, it selects the nearest slot to the entrance unit. It displays the slot number where the car is parked on the LCD. If user want to take back his vehicle he must swipe his car, then microcontroller will fetch the information and bring the vehicle to exit unit. Blended flag microcontrollers are normal, incorporating simple parts expected to control non-computerized electronic frameworks. With regards to the web of things, microcontrollers are an affordable and mainstream methods for information gathering, detecting and impelling the physical world as edge gadgets.

The automatic water sprinkler system has been implemented for additional security for fire detection with the help of buzzers.

The data analysis can be given as equations for fuel usage,time saving and Green House Gas(GHG) emissionreduction.

\section{Fuel Consumption:}

The fuel utilization every day for a computerized vehicle leaving framework can be determined utilizing the accompanying condition:

$F C=N S A$ ft

D

Where, FC $=$ fuel cost

$\mathbf{N}=$ Total number of cars parked and taken out per day $S=$ Speed of vehicle leaving plate A

$=$ Average distance traveled

D

\section{$f=$ Fuel consumption per unit distance}

$\mathbf{t}=$ Average time to park and to take out a car (in seconds)Time saving:

Time consumed in to park and retrieve a car is calculated by:

$$
T=T-T
$$

S M

$T=\left(D_{p} * t\right)+\left(D_{R} * t\right)+t e \ldots(3)$

Where, $\mathbf{T}=$ Time saved by automated car parking

system

$\mathbf{T}=$ Time devoured for robotized parking framework $T$

$$
\begin{aligned}
& =\text { Time expended for manual vehicle } \\
& \text { leaving }
\end{aligned}
$$

M

$D=$ Distance of slot from entrance $p$

$D=$ Distance of slot from exit

$\mathbf{R}$

$t=$ time to travel per unit distance $t$

$=$ Average time taken at exit gate $\mathrm{e}$

\section{GHG Emission:}

The decrease of GHG discharge rate can be determined by thefollowing formula:

$$
G=T_{S} * G_{R}
$$

\section{Where,}




\section{G = Diminished discharge of GHG}

RE

\section{T = Time saved; \\ G = Rate of GHG discharge per unit time}

The hardware requirements for the given system can be understood from the block digram. The proper functioning of the model is made through programming in embedded $\mathrm{C}$ using Keil software by mentioning proper delay and functions in the code.

\section{RESULTS}

In this system we have developed a hassle-free system thatwill be useful for traffic control and space consumption problems in smart cities.We can avoid congestion of unnecessary vehicles on the road which are parked illegally. In this system with advanced security features we can serveto provide efficient system which uses RFID identification.For this we have tested the authorisation using RFID cards with unique ID for different users.

\section{CONCLUSION}

The proposed system is designed using structured modeling and is able to provide the desired results. It very well may be effectively executed as a Real Time framework with specific changes. Science is finding or making real leap forward in different fields, and thus innovation continues changing every once in a while. Going further, a large portion of the units canbe manufactured on a solitary alongside microcontroller in this manner making the framework minimal in this waymaking the current framework progressively powerful. To make the framework pertinent for ongoing purposes parts with more prominent range should be executed.In this system, we have designed and implemented a Multi-storied IntelligentCar parking System using the concept of Embedded System. The target users of the system can be any one right from a common man to any organization.

\section{REFERENCES}

[1].Dr. V Kepuska and Humaid Al Hamsi, "smart car parking system"-International journal of science and Technology, Volume-5, Issue 14, pp. 390-395, No.8 August, 2016.

[2].J.Anitha,Y.Thoyajakshi,A.Ramya,V.Shravani,Prasha nt Kumar, "Intelligent Parking System using

Android

Application"International Journal of pure and applied mathematics,volume-114, pp-165-169 No. 7 April,2017.

[3].Rishi Anand,Abhirup Khanna- "IOT based Smart ParkingSystem"-International conference on Internet Of

Things and Applications(IOTA),pp-266-270, January, 2016

[4].Prabhu Ramaswamy- "IOT Smart Parking System for reducing green house gas emission"- Fifth International Conference On Recent Trends in Information Technology,pp-1-6, 2016.

This is an open access article under the CC-BY license. Know more on licensing on https://creativecommons.org/licenses/by/4.0/

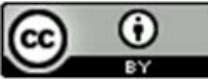

DOI - 10.55.83/irjeas.2022.v10i1003

Metadata from Crossref 